\title{
Correlation of 2D echocardiographic calcium score with severity of coronary artery disease assessed by C.T angiography and coronary angiography
}

K.E.E.El-Rabbat, A.I.Attia, H.H.Ebaid and M,E.Diab

cardiology, Dept., Faculty of Medicine, Benha Univ., Benha, Egypt

E-Mail: mohameddibo33@gmail.com

\begin{abstract}
Background: A major cause of mortality is coronary artery disease (CAD). The research evaluated the usefulness of 2D Echo calcium as a simple technique to predict the gravity of C.T. angiography and coronary angiography syntax. Methods; 100 patients of the coronary-computed tomography unit for coronary coronary CT were examined in this cross-sectional research and required coronary angiography. Patients have been exposed to histories, exams, blood samples and echocardiographic tests. The calcium echocardiography was linked to their coronary angiography and calcium CT scores syntactics. Results: There were 100 patients involved in the research, 68 of them were males representing $68 \%$ of the participants. The average age of the whole group was 53 . The mean final calcium echo score for the whole research group was 6 . The whole research group's mean syntactic score was 10. There was a significant difference in the overall ECHO calcium score between the three subgroups of the syntactic score and CT ( $\mathrm{p}$ value $<0.001$ both). Conclusion: The echocardiographic calcium score is linked with coronary artery disease severity and may thus be utilised as a novel tool for stratification of cardiovascular risks. The cheap cost, accessibility and radiation-free characteristics of echocardiography make it an appealing option for continuous development into non-invasive CAD prediction methods.
\end{abstract}

Keywords: calcium score, C.T, angiography, coronary, CAD.

\section{Introduction}

Coronary artery disease (CAD) is the world's leading cause of mortality. Coronary angiography is the golden standard for diagnosis and choice for the best treatment of coronary artery disease patients; however, efforts have been made to predict CAD severity and complexity by using non-invasive methods to identify those patients at high-risk for cardiovascular events at a lower risk to patients, and before coronary angiography. Coronary artery calcifier characterisation by computed tomography known as CAS is equal to the overall amount of coronary atherosclerosis and has been shown to be associated with angiographically significant damage [1].

A non-invasive technique to identify coronary artery disorders by detecting segmented wall movement anomalies and estimating the coronary calcium scores in aortic valve calcification (AVC), mitral anulus calcification (MAC), aortic root and papillary muscle calcification Dimensional Echocardiography [2].

A non-invasive approach for reliable CAD evaluation is coronary computed tomography angiography (CCTA). CCTA gives information about the vessel wall and the composition of plaques in addition to the degree of stenosis in contrast to invasive angiography (ICA) [3].

Coronary artery calcifications are closely linked with atherosclerosis, and a coronary artery calcium (CACS) angiography may be utilised to better predict the individual risk of future coronary events coronary artery calcifications [4].

Although CCTA is the reference technique for quantification of coronary and non-coronary heart calcium, echocardiography is considered to be a possible easy add-on to personalised risk prediction clinical scores if it is validated against CCTA [5].

The gold standard for the existence and / or severity of atherosclerotic coronary-artery disease remains coronary arteriography, helping to decide if coronary procedures are required [6].

Although alternative methods like as computed tomography (CT) and magnetic resonance imaging (MRI) have been developed, catheter-based selective, X-ray coronary angiography remains the most frequent way of precise imagery for the whole coronal tree, for a variety of reasons [6].

SYNTAX scoring (SS) is one CAD gravity and complexity evaluation method. SS includes lesion morphology, including complete occlusion, bifurcation, length, and lesion location on the basis of the at risk myocardial region [5].

The objective of the research was to evaluate the usefulness of 2D Echo calcium score as a simple method to forecast the severity of C.T angiographical and coronary angiography syntactic disease.

\section{Patients and methods}

This cross-sectional research was performed at Benha University Hospital \& Benha Health Insurance Hospital between March 2020 and April 2021. The patients are chosen for multislice coronary $\mathrm{CT}$ and require coronary angiography from the coronary computed tomography unit. Patients have been exposed to histories, exams, blood samples and echocardiographic tests.

The calcium echocardiography was linked to their coronary angiography and calcium CT scores syntactics. The syntactic score also has been split into three categories, with low risk in 18 , intermediate risk in 18-27 and high risks in 17 and $\mathrm{CT}$ in 3 groups; mild in 100 , moderate in 100 , severe in 400 , [7]. 
All the research patients received informed written permission. And the research was authorised by the Benha Faculty of Medicine Ethical Committee.

\subsection{Inclusion criteria}

Patient's age less than 65 years old was refered to do c.t coronary angiography.

\subsection{Exclusion criteria}

1. Age less than 18 and more than 65 .

2. Poor patient echogenicity.

3. Patients with renal failure or on hemodialysis.

4. Patients presented with ACS or cardiogenic shock.

5. Significant valvular heart disease, aortic aneurysm.

6. Patients with $\mathrm{AF}$ or frequent premature beats.

7. Patients have difficulties in performing $\mathrm{CT}$ as inadequate breath holding

\subsection{Standard trans-thoracic two dimensional echocardiographic examinations}

All patients were studied in the left lateral decubitus position by an cardiologist using an ultrasound system with an S3 transducer. Standard 2D and M-mode echocardiograms were obtained in the apical four-chamber, apical two-chamber, apical long axis and left parasternal views according to the American society of echocardiography and the European Association of Echocardiography guidelines in order to calculate [8].

1- By M-mode study:

- Left ventricular end diastolic dimension (LVEDD)

- Left ventricular end systolic dimension (LVESD)

- Inter ventricular septal diameter (IVSD)

- Left ventricular posterior wall diameter (LVPWD)

- Left ventricular ejection fraction (EF \%)

- Left ventricular fractional shortening (FS)

2- By 2-D Study:

- Assessment of left ventricular ejection fraction (EF \%) by modified simpson $(\mathrm{EF}=(\mathrm{EDV}-$ ESV)/EDV x 100). (9)

- Assessment of segmental wall motion abnormalities to exclude documented cases with CAD.

- To exclude any valvular lesion by colored Doppler.

- To asses cardiac calcium score through aortic valve calcification (AVC), mitral annulus calcification (MAC), aortic root and papillary muscle calcification.

\subsection{Coronary CT Angiography}

The extent and severity of CAD burden was measured by several coronary CT angiographic scores, including coronary artery calcium score (CACS). Coronary artery calcium was identified as a dense area in the coronary artery exceeding the threshold of $130 \mathrm{HU}$. An overall Agatston score was recorded for each patient. The patient were classified into three groups according to calcium score: (10)

- Mild group : $<100$

- Moderate group : $\geq 100<400$

- Sever group : $\geq 400$

\subsection{Cardiac catheterization:}

1. A team of experienced intervention cardiologists conducted coronary angiography. The operators conducted a thorough examination of angiographical pictures.

2. Significant lesion is defined as $70 \%$ or more luminous stenose of any major coronary epicardial artery, and $50 \%$ or more in the left main coronary artery.

3. A visual estimate is used to evaluate the existence of severe lesions. Basal angiographical features are documented in patients such as the sick vascular, the left major coronary arters (LMs), the left anterior coronary artery; the right coronary artery (RCA).

4. Syntax score has been calculated using dedicated software (version 2.11, www.syntaxscore.com), the acceptable core lab reproductivity of the SYNTAX score, which has been reported to integrate two components; - morphological characteristics of each lesions like dominance, total chronic occlusion (CTO).

os Lesion weighting factors depending on the distal myocardial area to the lesion. SS is provided with lesions with 50 percent luminal blockage in vessels with a diameter of $1,5 \mathrm{~mm}$.

$\S$ All morphological characteristics of each SS injury have been documented.

- The SS has been split into three tertiles: low to 18 , moderate risk 18 to 27 and high risk groups $>27$.

\subsection{Analysis of statistics}

The SPSS version 25 was used for data management and statistical analysis (IBM, Armonk, New York, United States). Using the KolmogorovSmirnov test, the Shapiro Wilk test and direct data visualisation techniques, quantitative data were evaluated for normalcy. The numerical data were reported as means and standard deviations or mediums and ranges as per the usual tests. Numbers and percentages were summarised as categorical data. The quantitative data were compared by syntax and CT ca scores using one-way ANOVA or Kruskal Wallis, respectively for numerical variables that are normally or non-normally distributed. Categorical data have been compared using the Chi-square test or the exact test of Fisher, where applicable. All post hoc analyses were corrected by Bonferroni. The correlation study was carried by utilising the correlation of Pearson. After each of the test statistics has been calculated, the appropriate distribution tables have been checked for "P" (probability value). 
The $\mathrm{P}$ value $<0.05(\mathrm{~S})$ and a $\mathrm{P}$ value $<0.001$ were deemed very significant statistical significance (HS). At the same time, a $\mathrm{P}$ value $>0.05$ was not deemed significant (NS).

\section{Results}

The average patient age was $53 \pm 7$ years. Gender was mostly male; (68 percent) were male. In addition, diabetes, hypertensives, fume patients $(68 \%$, $44 \%, 44 \%$ ) and only $12 \%$ demonstrated favourable family history. The mean systolic and diastolic blood pressure was 129 and 78 . The mean cardiac rate was $70 \mathrm{~b} / \mathrm{m}$. The average weight and height respectively were 89 and 179. The mean BMI was 27.9, (Table 1).

LVIDd was significantly lower in the intermediate-risk group (40.5) compared to the low (48.3) and high risk (53.8) groups (P-value <0.001).

LVPWd was significantly higher in the low-risk group (11.1) compared to the high-risk group (7.6). (P-value < 0.001)

ESV was significantly lower in the intermediaterisk group (38.3) than the high-risk group (53.2). (Pvalue $=0.027)$.
EF was significantly higher in the low-risk group (63) than the high-risk group (54). (P-value = $0.006)$.

RWMA was significantly lower in the low-risk group $(20.0 \%)$ than intermediate $(60.0 \%)$ and high $(54.5 \%)$ risk groups, respectively. $(\mathrm{P}$-value $=0.002)$.

Diastolic dysfunction was significantly different in the low-risk group from the high-risk group. (Pvalue $=0.002)$. Grade I was $(95.0 \%, 80.0 \%, 72.7 \%)$ in lowe risk, intermediate risk \& high risk ,respectively.Grade II was ( $0.0 \% 10.0 \%, 27.3 \%)$ in lowe risk, intermediate risk $\&$ high risk, respectively

No significant differences were noted regarding IVSd, LVIDs, and EDV. Table (2).

One-way ANOVA was used for numerical data. Chi-square or Fisher's exact test was used for categorical data. Post hoc was done in case of significant overall effect, and different letters indicate significant pair,EDV; End diastolic volume ESV; End systolic volume ,EF; Ejection fraction ,RSWMA; resting segmental wall motion abnormality

Table (1) General characteristics of the studied patients.

\begin{tabular}{|c|c|c|}
\hline \multicolumn{3}{|l|}{ General characteristics } \\
\hline Age (years) & Mean \pm SD & $53 \pm 7$ \\
\hline \multirow{2}{*}{ Gender } & Males & $68(68.0)$ \\
\hline & Females $n(\%)$ & $32(32.0)$ \\
\hline Diabetes mellitus & n $(\%)$ & $68(68.0)$ \\
\hline Hypertension & n $(\%)$ & $44(44.0)$ \\
\hline Smoking & n $(\%)$ & $44(44.0)$ \\
\hline Family history & n $(\%)$ & $12(12.0)$ \\
\hline Systolic blood pressure & Mean \pm SD & $129 \pm 12$ \\
\hline Diastolic blood pressure & Mean \pm SD & $78 \pm 10$ \\
\hline Heart rate & Mean \pm SD & $70 \pm 12$ \\
\hline Weight (kg) & Mean \pm SD & $89 \pm 8$ \\
\hline Height $(\mathbf{c m})$ & Mean \pm SD & $179 \pm 7$ \\
\hline Body mass index & Mean \pm SD & $27.9 \pm 2.9$ \\
\hline
\end{tabular}

Correlation between Echo parameters \& syntax score

Table (2) Correlation between Echo parameters \& syntax score.

\begin{tabular}{|c|c|c|c|c|c|c|}
\hline & & & $\begin{array}{c}\text { Low risk } \\
(\mathrm{n}=\mathbf{4 0})\end{array}$ & $\begin{array}{c}\text { Syntax Score } \\
\text { Intermediate risk } \\
(\mathbf{n}=\mathbf{2 0})\end{array}$ & $\begin{array}{c}\text { High risk } \\
(\mathrm{n}=\mathbf{2 2})\end{array}$ & P-value \\
\hline IVSd & Mean \pm SI & & $10.1 \pm 1.3$ & $9.6 \pm 3.9$ & $8.5 \pm 4.2$ & 0.153 \\
\hline LVIDd & Mean \pm SI & & $48.3 \pm 8.3^{\mathrm{a}}$ & $40.5 \pm 13.4^{b}$ & $53.8 \pm 7^{\mathrm{a}}$ & $<0.001$ \\
\hline LVPWd & Mean \pm SI & & $11.1 \pm 1.8^{\mathrm{a}}$ & $9.6 \pm 3.3^{\mathrm{a}, \mathrm{b}}$ & $7.6 \pm 4.1^{b}$ & $<0.001$ \\
\hline LVIDs & Mean \pm SI & & $29.1 \pm 9.3$ & $28.2 \pm 10.6$ & $33.3 \pm 12.4$ & 0.221 \\
\hline EDV & Mean \pm SI & & $113 \pm 25.2$ & $98 \pm 19.5$ & $115.2 \pm 38$ & 0.096 \\
\hline ESV & Mean \pm SI & & $43 \pm 15.9^{a, b}$ & $38.3 \pm 12.3^{\mathrm{a}}$ & $53.2 \pm 25.4^{b}$ & 0.027 \\
\hline Ef simpson (\%) & Mean \pm SI & & $63 \pm 7^{\mathrm{a}}$ & $61 \pm 11^{\mathrm{a}, \mathrm{b}}$ & $54 \pm 14^{\mathrm{b}}$ & 0.006 \\
\hline RSWMA & n $(\%)$ & & $8(20.0)^{\mathrm{a}}$ & $12(60.0)^{\mathrm{b}}$ & $12(54.5)^{b}$ & 0.002 \\
\hline $\begin{array}{l}\text { Diastolic } \\
\text { dysfunction }\end{array}$ & $\begin{array}{l}\text { Normal } \\
\text { Grade I } \\
\text { Grade II } \\
\end{array}$ & $\begin{array}{l}\text { n }(\%) \\
\text { n }(\%) \\
\text { n }(\%)\end{array}$ & $\begin{aligned} 2 & (5.0) \\
38 & (95.0) \\
0 & (0.0)\end{aligned}$ & $\begin{array}{c}2(10.0) \\
16(80.0) \\
2(10.0) \\
\end{array}$ & $\begin{array}{c}0(0.0) \\
16(72.7) \\
6(27.3) \\
\end{array}$ & 0.002 \\
\hline
\end{tabular}


Table (3) Echo calcium score according to syntax score.

\begin{tabular}{|c|c|c|c|c|c|}
\hline & & $\begin{array}{c}\text { Low risk } \\
(\mathrm{n}=40)\end{array}$ & $\begin{array}{c}\text { Syntax Score } \\
\text { Intermediate risk } \\
(\mathrm{n}=\mathbf{2 0})\end{array}$ & $\begin{array}{l}\text { High risk } \\
(\mathrm{n}=\mathbf{2 2})\end{array}$ & P-value \\
\hline Papillary calcification & Median (range) & $1(0-1)$ & $1(1-1)$ & $1(1-1)$ & 0.345 \\
\hline Mitral Annulus calcification & Median (range) & $2(1-3)^{a}$ & $2(2-3)^{a, b}$ & $3(2-3)^{b}$ & 0.001 \\
\hline Aortic root calcification & Median (range) & $1(0-1)$ & $1(1-1)$ & $1(1-1)$ & 0.345 \\
\hline Aortic valve calcification & Median (range) & $2(1-3)^{a}$ & $3(2-3)^{b}$ & $3(2-3)^{b}$ & $<0.001$ \\
\hline Echo calcium score & Median (range) & $6(4-8)^{a}$ & $7(6-8)^{b}$ & $8(6-8)^{b}$ & $<0.001$ \\
\hline
\end{tabular}

Kruskal Wallis test was used. Post hoc was done in case of significant overall effect, and different letters indicate significant pair

\section{Correlation between Echo calcium score \& syntax score}

- Mitral annulus calcification was significantly lower in the low-risk group (2) than the high-risk group $(3)$. $(\mathrm{P}$-value $=0.001)$.

- Also, Aortic valve calcification was significantly lower in the low-risk group (2) than the intermediate (3) and high (3) risk groups. (Pvalue $<0.001$ ).

- Echo calcium score was significantly lower in the low-risk group (6) compare to the intermediate (7) and high (8) risk groups. (Pvalue $<0.001)$.

No significant differences were noted regarding papillary calcification and aortic root calcification. Table (3)

\section{Discussion}

The present research consisted of 100 patients chosen from the multislice coronary CT coronary tomography unit and required coronary angiography. They gathered and statistically evaluated their gender, risk factors, laboratory data and angiographical findings. In our research, the syntactic score and CT coronary angiography were employed as an indication of the severity of coronary artery disease and associated with the various elements of echocary calcium score.

The patients have been categorised in three categories based on their angiographical findings and syntactic values: low risk group 18, middle risk group 18-27, and high risk groups $>27$.

Obviously there was a significant difference between LVPWd, ESV, EF, RWMA, diastolic dysfunction p-value $(<0.001,<0.001,0.027,0.006$, $0.002,0.002)$, and the similar results of Mostafa and others with respect to LVEDD \& diastolic dysfunction showed only that the highest SYNTAX score was significantly related with LVEDd and LVID (11).

\subsection{Correlation between the echo calcium score and the syntax score:}

Also, in the prediction of the severe coronal artery diseases similar to the results of Suhas Hardas and others, our study found a significant correlation between the final calcium score by echocars and syntax score subgrups with a p <0.001 value. (2) Significant correlations between echocardiographic calcium score and CAD were found by Corciu et al. Echocardiographic calcium score was evaluated as a calcium scores index (CSI) that did not include papillary muscle calcification as part of the score, and coronary angiography(duke scores) that split its study population into two subgroups evaluated the existence of CAD. It also revealed a substantial rise in the mean CSI with the presence of impacted pvalue 0.002 coronaries. (12) Corciu et al also found substantial correspondence between each component of the CSI and the p CAD value 0.001 for AR, 0.022 for MAC and 0.013 for the calcification of the aortic valve. In our research MAC, there were significant differences in the levels of syntax of aortic root calcification (P-value of $0,001,<0,001$ ), but no significant differences in papillary calcifications and aortic root calcification were observed (12).

\subsection{Regarding Echo calcium score and CT calcium score correlation}

The study found a strong correlation in the calcium score by echocardiography to the calcium score subgroups with $(\mathrm{r}=0.640, \mathrm{p}<0.001)$ similar to a number of studies, including Gaibazzi et al(5) \& Samar et al. (13), which also reported positive correlating between echo calcium score and high CACS detected by MSCT with $\mathrm{p}$ value of 0.0026 for Gaibazzi.

Utsunomiya et al. further investigated the combined presence value of aortic valve calcification and mitral annular calcification as an indicator of the size and vulnerability of coronary artery plaque evaluated in 64-multidetector computed tomography and validated the earlier findings in this respect (14).

Perssman et al. also found the connection between calcium and CAD echocardiography. 
The calcium echocardiography score included various components and CAD was assessed in CCTA as CAC. There was a significant connection between the two with a value of $\mathrm{p} 0.0024$. It has been shown that overall cardiac calcium is better for CAD than MAC alone without commenting on other calcium score components. While in our study the final calcium score was correlated significantly to the CT calcium subgroups $(<0.001)$, there was a significant correlation to the papillary calcification, mitral annulus calcification, aortic p-value calcification $(0.005,<0.001,<0.001)$, respectively, without a sign (15).

Concerning the correlation between $\mathrm{CC}$ and syntax score, Shabbier et al.' found a positive correlation between the score of syntax and the score of ct calcium $(\mathrm{r}=0.354, \mathrm{p}=0.001)$ and confirmed results Syntax score showed a significant positive correlation with the score of CT calcium $(r=0.785 \&$ CT < 0.001) (16).

\section{Conclusion}

Echocardiographic calcium results are related to the degree of coronary artery disease and thus may be utilised as a novel instrument for the classification of cardiovascular risk. The cheap cost, accessibility and radiation-free characteristics of echocardiography make it an appealing option for continuous development into non-invasive $\mathrm{CAD}$ prediction methods.

\section{Financing sources}

This study received no particular grant from governmental, commercial or non-profit funding organisations.

\section{Contribution to the author}

The authors also contributed to the research.

\section{Interest Conflicts}

No interest disputes.

\section{References}

[1] K.Nasir, M.Clouse Role of nonenhanced multidetector CT coronary artery calcium testing in asymptomatic and symptomatic individuals.

Radiology.vol.264,pp.63749,2012.

[2] S.Hardas,P.Titar,I.Zanwar, DS.Phalgune Correlation between echocardiographic calcium score and coronary artery lesion severity on invasive coronary angiography. Indian Heart J.vol.22,pp.120-123,2021.

[3] MJ.Budoff, D.Dowe, JG.Jollis, M.Gitter, J.Sutherland, E.Halamert. Diagnostic performance of 64-multidetector row coronary computed tomographic angiography for evaluation of coronary artery stenosis in individuals without known coronary artery disease: results from the prospective multicenter ACCURACY (Assessment by Coronary Computed Tomographic Angiography of Individuals Undergoing
Invasive Coronary Angiography) trial. J Am Coll Cardiol.vol.52,pp.1724-32,2008.

[4] MC.Williams, J.Kwiecinski, M.Doris, P. McElhinney, MS.D'Souza, S.Cadet. Lowattenuation noncalcified plaque on coronary computed tomography angiography predicts myocardial infarction: results from the multicenter SCOT-HEART trial (Scottish Computed Tomography of the HEART). Circulation.vol.141,pp.1452-62,2020.

[5] N.Gaibazzi, C.Baldari, P.Faggiano, L.Albertini, G.Faden, F.Pigazzani. Cardiac calcium score on 2D echo: correlations with cardiac and coronary calcium at multi-detector computed tomography. Cardiovasc Ultrasound.vol.12,pp.1-9,2014.

[6] C.Di Mario, N.Sutaria Coronary angiography in the angioplasty era: projections with a meaning. Heart.vol.91,pp.968-76,2005.

[7] JY.Cho, KH.Kim Evaluation of arterial stiffness by echocardiography: methodological aspects. Chonnam Med J.vol.52,pp.1016,2016.

[8] C. Mitchell, PS .Rahko, LA .Blauwet, B .Canaday, JA. Finstuen, MC .Foster.Guidelines for performing a comprehensive transthoracic echocardiographic examination in adults: recommendations from the American Society of Echocardiography. J Am Soc Echocardiogr.vol.32,pp.1-64,2019.

[9] R. Votavová, A. Linhartová, J .Kořínek, J .Marek, A. Linhart. Echocardiography in coronary artery disease. Cor Vasa.vol.57,pp.e408-18. ,2015.

[10] AS .Agatston, WR .Janowitz, FJ .Hildner, NR. Zusmer, M .Viamonte, R.Detrano Quantification of coronary artery calcium using ultrafast computed tomography. J Am Coll Cardiol.vol.15,pp.827-32,1990.

[11]MM.Abdelrazik, EED.El Sawy, AM.Fahmy, F.Rafik Correlation between dyslipidiemia and the severity of coronary artery disease using syntax scoring system. Egypt J Hosp Med.vol.66,pp.52-6,2017.

[12] AI.Corciu, V.Siciliano, E.Poggianti, C.Petersen, L.Venneri, E.Picano Cardiac calcification by transthoracic echocardiography in patients with known or suspected coronary artery disease. Int J Cardiol.vol.142,pp.288-95,2010.

[13] SA. Nasreldeen, BMA.Hady, OH.Abd Elaziz, AM.Ahmed Value of 2D echo calcium score and neutrophil/lymphocyte ratio as simple tools for the prediction of coronary artery disease documented by multislice computed tomography in type II diabetic patients. Sci J Al-Azhar Med Fac Girls.vol.4,pp.439446,2020 . 
[14]H .Utsunomiya, H.Yamamoto, E.Kunita, T.Kitagawa, N.Ohashi, T.Oka.. Combined presence of aortic valve calcification and mitral annular calcification as a marker of the extent and vulnerable characteristics of coronary artery plaque assessed by 64multidetector computed tomography. Atherosclerosis. .vol.213,pp.166-72,2010.

[15] GS.Pressman, V.Crudu, A.ParameswaranChandrika, A.Romero-Corral, B. Purushottam,
VM.Figueredo Can total cardiac calcium predict the coronary calcium score? Int $\mathbf{J}$ Cardiol.vol.146,pp.202-6,2011.

[16] A.Shabbir, ST.Virk, J.Malik, S.Kausar, TB.Nazir, A.Javed Coronary Artery Calcium Score: Assessment of SYNTAX Score and Prediction of Coronary Artery Disease. Cureus.vol.13,pp.149-170,2021. 